liminary results, the company plans to move forward with phase 2 trials.

\section{Noting risk}

Generic drug companies are not responsible for updating the warning labels on their products; however, the US Food and Drug Administration (FDA) proposed a regulatory update on 27 March that would compel these companies to do so. Currently, generic drugs-which constitute $80 \%$ of filled prescriptions - update their warning labels only if specifically mandated by the FDA after their brand-name counterparts update the warning label on the corresponding medication. Many generic drug companies are challenging this new plan, arguing that it would increase costs, as they would have to collect their own information from patients using their versions instead of relying on data collected by brand-name manufacturers. "The proposed rule is critical for filling a safety gap that exists today, says Michael Carome, director of the health research group at the Washington, DC-based consumer rights advocacy group Public Citizen. "It would give generic drug companies the incentive and authority to monitor and update their labels, ultimately protecting patients and improving patient safety."

\section{Generic greenlight}

The FDA gave its approval on 16 April to the first generic version of Copaxone (glatiramer acetate), a commonly used drug for the treatment of relapsing multiple sclerosis. The agency approved a generic version called Glatopa, made by German genericsmaker Sandoz and Cambridge, Massachusetts-based Momenta Pharmaceuticals. Copaxone is Israeli drug giant Teva Pharmaceuticals's biggest product, accounting for more than $\$ 4$ billion in annual revenue, which is nearly $21 \%$ of its overall revenue. The generic is based on an older version of Copaxone, which is a less-concentrated version of the current form of the drug, requiring daily injections instead of the current dose of three times a week. Sandoz and Momenta have not released details about how much cheaper Glatopa will be compared to Copaxone, nor have they set a market release date.

\section{POLICY}

\section{Noninvasive screens}

On 24 March, two of the world's largest professional societies of geneticists, the European Society of Human Genetics and the American Society of Human Genetics, published a statement on the promises and challenges of noninvasive prenatal testing (NIPT) (Eur. J. Hum. Genet., doi: 10.1038/ ejhg.2015.57, 2015). In their statement, the societies asserted that NIPT, which analyzes fragments of DNA in a mother's blood for abnormalities in her child, could replace or supplement current invasive prenatal screening methods such as amniocentesis. The authors also added that although NIPT could test for chromosomal problems such as aneuploidy, it might be too early to routinely apply the approach to test for smaller genetic changes linked to disease. "[NIPT] can become the first aneuploidy screening test for many women and reduce the number of invasive procedures," says Martina Cornel, an author of the report. "NIPT needs to be integrated into the practices of midwives and obstetricians. Public health authorities could support this at a national level."

\section{Fighting resistance}

On 27 March, President Obama asked the US Congress to allocate $\$ 1.2$ billion toward fighting antibiotic resistance. This national strategy will include improved surveillance of antibiotic use and outbreaks, better diagnostic tests and research for the discovery of novel antibiotics. The national plan aims to reduce inappropriate antibiotic use in the outpatient setting by $50 \%$, and to reduce the time it takes to detect antibiotic-resistant infections by $25-50 \%$ by 2020 . "We need nothing short of an 'all hands on deck' effort" to combat antibiotic resistance, says Anthony So, director for global health at Duke University's School of Public Policy in Durham, North Carolina. "The roadmap laid out by the government provides a promising starting point to support antibiotic stewardship efforts."

\section{Expedited pathway}

On 15 April, a year after the idea was formally proposed, the FDA began accepting applications beginning for the accelerated approval program for critical medical devices called the Expedited Access Pathway (EAP), which was finalized two days earlier. The program applies to devices that demonstrate the potential to address unmet medical needs for life-threatening or irreversibly debilitating diseases or conditions. To be accepted in the program, a device must treat or diagnose a condition for which there are no current tools, or provide an advantage over existing technologies. "The FDA is committed to ensuring that patients have timely access to medical devices that are safe and effective," says Leslie Wooldridge, a spokeswoman at the FDA. "The EAP allows us to work with manufacturers much earlier in the process to outline a plan that balances data collection." The EAP will reduce the clinical data requirements currently required for devices by using surrogate endpoints that are easier and faster to measure than traditional clinical endpoints.

\title{
Corrections
}

An earlier version of the article "New technologies take root in the search for antibiotics from soil" (Nat. Med. 21, 201, 2015) inaccurately stated that researchers have not identified any new molecules using iChip technology. In fact, this technology has found new molecules and genes of interest. The error has been corrected in the HTML and PDF versions of the article.

The version of the "An unknown enemy: Drugs sought against EV-68 as paralysis link is explored" article initially published online on 30 March 2015 (Nat. Med. doi:10.1038/nm.3846, 2015) failed to note Michael Rossmann's affiliation or the involvement of his collaborators, and it incorrectly placed Emory University in Decatur, Georgia, when in fact it is in Atlanta. The errors have been corrected in the HTML and PDF versions of the article.

In the version of the article "Warren joins lawmakers proposing new ideas for science funding" initially published, (Nat. Med. 21, 299, 2015), the legislation recently introduced by Senator Warren was oversimplified, in a manner that implied it would increase the amounts of fines paid by pharmaceutical companies via settlement agreements with the Department of Justice. Rather than directly affect these settlement amounts, the Medical Innovation Act would also force companies that have entered into settlement agreements with the Department of Justice for breaking federal laws to pay an additional $1 \%$ of their total profits for each blockbuster drug that can be traced back to government research support, for a period of five years. The supplemental payments would go directly to biomedical research rather than the general treasury, increasing science funding without requiring a new tax. The error has been corrected in the HTML and PDF versions of the article. 